\title{
Comparative study of spectroscopic properties of some indium, tin and antimony compounds
}

\author{
by G. IGEL-MANN, C. FELLER, H.-J. FLAD, A. SAVIN, \\ H. STOLL and H. PREUSS \\ Institut für Theoretische Chemie, Universität Stuttgart, \\ Pfaffenwaldring 55, 7000 Stuttgart 80, F.R. Germany
}

(Received 18 April 1989; accepted 8 May 1989)

\begin{abstract}
Spectroscopic properties of dimers, hydrides, oxides, fluorides and sulphides of the elements $\mathrm{In}, \mathrm{Sn}$ and $\mathrm{Sb}$ have been calculated using energy-adjusted pseudopotentials. Results are given for bond lengths $\boldsymbol{R}_{e}$, dissociation energies $D_{e}$, vibrational frequencies $\omega_{e}$ and dipole moments $\mu_{e}$ of the ground states. Comparison is made both with experimental and theoretical values, where available. The influence of core-core overlap, of valence correlation as well as of core-valence correlation is discussed. For $\mathrm{In}_{2}$, three low-lying states have been investigated. Contrary to experimental results, the ground state of $I n_{2}$ is found to be ${ }^{3} \mathrm{\Pi}$. Ionization potentials of $\mathrm{In}, \mathrm{Sn}, \mathrm{Sb}$ and electron affinities of $\mathrm{O}$, $\mathrm{F}, \mathrm{S}$ have also been calculated.
\end{abstract}

\section{Introduction}

Theoretical investigations on molecules become rather rare with increasing atomic number of the elements because of the drastic increase of computational effort. This effort can be reduced by using pseudopotentials [1-5]. Only the valence electrons are treated explicitly, while the core is represented by a pseudopotential. In addition, relativistic effects become more and more important with increasing atomic number. In contrast to relativistic all-electron calculations, which, for molecules, are mostly restricted to the Dirac-Fock (DF) level up to now (for a review see [6]), relativistic effects can be included into pseudopotentials in a simple way. Therefore, the pseudopotential method seems to be the most successful molecular theory for heavy atoms [7]. Further merits of the pseudopotential method are that there is no significant loss in accuracy, and that all elements of the same group of the Periodic Table can be treated on equal footing.

For the main group elements $\mathrm{In}, \mathrm{Sn}$ and $\mathrm{Sb}$, pseudopotentials have been published by several authors [8-14]. Instead of the adjustment to orbital energies and orbital densities [8-13], the pseudopotentials used in this work [14] are fitted to experimental ionization and excitation energies of one-valence electron atoms [15]. This treatment implicitly includes relativistic effects. Core-valence correlation can easily be introduced by means of an additional core-polarization potential [16] without extension both of the valence shell to $d$ orbitals and of the basis set to $f$ functions.

Applications of our pseudopotentials to various main group element compounds have been published in the last few years [17-25]. Deviations from reliable experimental data are almost negligible for alkali compounds $[18-20,22]\left(\Delta R_{e}=0.03 \AA\right.$ and $\Delta D_{e}=0.02 \mathrm{eV}$ ), but increase for hydrides and oxides [24] of fourth-row elements up to $\Delta R_{e}=0.05 \AA$ and $\Delta D_{e}=1.1 \mathrm{eV}$, respectively, mainly due to size- 
consistency errors in the treatment of valence correlation. Application is now made to molecules containing the fifth-row elements In, Sn and Sb.

In recent papers, compounds of group IVA elements with group IA [23] and group IB elements [25] have been investigated. Several structures of these molecules $A_{n} B_{m}(A=\mathrm{Si}, \mathrm{Ge}, \mathrm{Sn} ; B=\mathrm{Li}, \mathrm{Cu} ; n=m=2,4)$ have been discussed. The influence of the inert electron pair of the group IVA elements on the bonding has been considered. In the near future, we plan to extend these investigations to closed-shell molecules $\left(X H_{m}\right)_{n}$ and $\left(X Y_{m}\right)_{n}(X=\mathrm{In}, \mathrm{Sn}, \mathrm{Sb} ; Y=\mathrm{O}, \mathrm{F}, \mathrm{S} ; n \leqslant 6)$. The behaviour of the inert electron pairs will be of interest, especially in the latter case where both the $X$ and $Y$ atoms contain such pairs. To keep the computational effort within reasonable limits, rather small basis sets have to be used. Therefore, in the present paper, the accuracy of our pseudopotential method and the basis sets used will be checked for spectroscopic properties of the diatomic molecules $X Y$ and, additionally, of the homonuclear dimers $X_{2}(X=\mathrm{In}, \mathrm{Sn}, \mathrm{Sb} ; Y=\mathrm{H}, \mathrm{O}, \mathrm{F}, \mathrm{S})$. The influence of several effects on bond lengths $R_{e}$, dissociation energies $D_{e}$ and vibrational frequencies $\omega_{e}$ is determined using two different basis sets. In particular, the effect of core-core interaction and both valence and core-valence correlation is considered. Valence correlation effects have been determined by means of several different methods. At the highest level of approximation, comparison of the properties calculated with both basis sets is made with experimental values. Furthermore, dipole moments $\mu_{e}$ are given. Comparison is made with other theoretical results where available. Since diatomic molecules $X Y(X=\mathrm{In}, \mathrm{Sn}, \mathrm{Sb} ; Y=\mathrm{O}, \mathrm{F}, \mathrm{S})$ are strongly ionic, we have determined atomic properties, additionally: for the atoms In, $\mathrm{Sn}, \mathrm{Sb}$ and $\mathrm{O}, \mathrm{F}, \mathrm{S}$, ionization potentials and electron affinities, respectively, are compared with numerical Hartree-Fock (HF) and experimental values.

\section{Method}

In our calculations, we use a valence model hamiltonian (in atomic units)

$$
H_{\text {mod }}=-\frac{1}{2} \sum_{i} \Delta_{i}+V_{s p}+\sum_{i<j} \frac{1}{r_{i j}}+\sum_{\lambda<\mu} \frac{Q_{\lambda} Q_{\mu}}{r_{\lambda \mu}}
$$

( $i, j$ denote valence electrons; $\lambda, \mu$ are core indices and $Q_{\lambda}, Q_{\mu}$ are core charges). $V_{s p}$ is a semilocal pseudopotential

$$
V_{s p}=-\sum_{\lambda, i} \frac{Q_{\lambda}}{r_{\lambda i}}+\sum_{\lambda, i} \sum_{i, j} B_{l j}^{\lambda} \exp \left(-\beta_{l j}^{\lambda} r_{\lambda i}^{2}\right) P_{l}^{\lambda}+V_{\mathrm{pol}}
$$

$P_{l}^{\lambda}$ is the projection operator on angular momentum $l$ with respect to core $\lambda . V_{\text {pol }}$ is a core-polarization potential of the type

$$
V_{\text {pol }}=-\sum_{\lambda} \frac{1}{2} \alpha_{\lambda} \mathbf{f}_{\lambda}^{2}
$$

with

$$
\mathbf{f}_{\lambda}=\sum_{i} \mathbf{r}_{\lambda i} r_{\lambda i}^{-3}\left(1-\exp \left(-\delta_{\lambda} r_{\lambda i}^{2}\right)\right)-\sum_{\mu(\neq \lambda)} Q_{\mu} \mathbf{r}_{\lambda \mu} r_{\lambda \mu}^{-3}
$$

Here $\alpha_{\lambda}$ is the dipole polarizability of core $\lambda$ and $\mathbf{f}_{\lambda}$ is the field produced at the site of core $\lambda$ by the valence electrons and other cores. The cut-off factor (with parameter $\delta_{\lambda}$ ) in equation (4) and the parameters $B_{l j}^{\lambda}, \beta_{l j}^{\lambda}$ in equation (2) are adjusted to 
single-valence-electron atomic data: to experimental and Dirac-Fock (DF) values of the first ionization potential, and to experimental excitation energies of the valence electron. As the core-polarization potential is explicitly included in our semilocal pseudopotential $V_{s p}$, frozen-core DF pseudopotentials $\left(V_{\mathrm{DF}}\right)$

$$
V_{\mathrm{DF}}=V_{s p}-V_{\mathrm{pol}},
$$

are easily available without changing the pseudopotential parameters (cf. equation (2)) [26]: due to our adjustment, relativistic effects are implicitly included in $V_{\mathrm{DF}}$. Details of the fitting procedure are described in [26]. The pseudopotential parameters used in this work have already been published [14].

In this work, atomic and molecular spectroscopic properties have been determined. Valence SCF calculations with the model hamiltonian (cf. equation (1)) have been performed. For $V_{\mathrm{DF}}$ and $V_{s p}$, the programs MOLPRO [27] and MELD [28] modified by our group have been used, respectively. Correlation energies have been calculated by means of complete active space SCF (CASSCF), spin density functional (SDF) and CI calculations with all single and double excitations from the SCF reference determinant (SD-CI). In the latter case, the contribution of quadruple excitations has been estimated by means of Davidson's correction [29] (SD$\mathrm{CI}+Q$ ) in order to reduce size-consistency errors. Our gaussian basis sets (GTO) used in all cases are (i) $(6 s 6 p 1 \mathrm{~d}) /[3 s 3 p 1 d]$ for $X(X=\mathrm{In}, \mathrm{Sn}, \mathrm{Sb})$ and $(6 s 7 p 2 d 1 f) /$ $[3 s 4 p 2 d 1 f]$ for $Y(Y=\mathrm{O}, \mathrm{F}, \mathrm{S})$ (basis set I) and (ii) $(4 s 4 p 1 d) /[2 s 2 p 1 d]$ for $X(X=\mathrm{In}$, $\mathrm{Sn}, \mathrm{Sb})$ and $(4 s 5 p 1 d) /[2 s 3 p 1 d]$ for $Y(Y=\mathrm{O}, \mathrm{F}, \mathrm{S})$ (basis set II). (Basis set I has been augmented by $d$ and $f$ functions on the $Y$ atoms for a better description of the $Y^{-}$anions.) For $\mathrm{H}$, a $(5 s 1 p) /[2 s 1 p]$ basis set has been used [30]. The $s$ and $p$ exponents for the $X$ atoms $(X=\mathrm{In}, \mathrm{Sn}, \mathrm{Sb})$ and the $Y$ atoms $(Y=\mathrm{O}, \mathrm{F}, \mathrm{S})$ have been energy-optimized in SCF calculations for the atomic ground states using the program PSATOM [31], whereas the $d$ and $f$ exponents have been optimized in atomic SD-CI calculations for $X^{+}$and $Y^{-}(X=\mathrm{In}, \mathrm{Sn}, \mathrm{Sb} ; Y=\mathrm{O}, \mathrm{F}, \mathrm{S})$.

For the atomic calculations, numerical HF values are taken as references, both all-electron quasirelativistic (AE) and pseudopotential (PP) ones which have been determined by means of the program MCHF77 [32] modified by our group. (The quasirelativistic $\mathrm{HF}-\mathrm{AE}$ values include a mass-velocity term and a Darwin (averaged) spin-orbit term in the form suggested by Wood and Boring [33].)

For the molecular calculations, we have investigated several levels of approximation in this paper which will be explained in the following. At the lowest level $(A)$, valence SCF calculations with $V_{\mathrm{DF}}$ have been done using the program MOLPRO [27] and our gaussian basis set I. In the next step $(B)$, deviations of the core-core interaction from the point-charge approximation are taken into account as described in detail in [26]. Effects of changing to the smaller basis set II are described at level $C$. A higher level of approximation is reached by including valence correlation which we have determined in this work by (i) CASSCF (level $D$ ), (ii) SDF (level $E$ ) and (iii) CI calculations including all single and double excitations from the SCF reference determinant (SD-CI) (level $F$ ). (For SDF, various density functionals have been taken into account, e.g. with self-interaction correction [34, 35], with gradient correction [36] and with second-order gradient correction [37]. We cannot give a clear preference to one of the density functionals considered because, for the molecules investigated in this paper, all three functionals lead to similar results. Therefore, only results with one of them, the gradient corrected functional by Perdew [36], have been tabulated.) To reduce size-consistency errors, the SD-CI 
results have been corrected by contributions of quadrupole excitations [29] (level $G$ ). At this point, comparison with results obtained with basis set $\mathbf{I}$ (level $H$ ) is made again. At the highest level of approximation $(I)$, core-valence correlation is taken into account by introducing our core-polarization potential $V_{\text {pol }}$ yielding the pseudopotential $V_{s p}$ (cf. equation (2)). (For these calculations, the program MELD [28] has been used because core-polarization is not implemented in the program MOLPRO [27] at the moment.) At this level, comparison with experimental data $(K)$ is made.

Bond lengths $R_{e}$, dissociation energies $D_{e}$ and vibrational frequencies $\omega_{e}$ have been determined by calculating (total) valence energies at four or five points around the minimum of the potential curve and fitting these values to third-degree polynomials. At the calculated equilibrium geometry, we have determined dipole moments which are corrected, in the case of $V_{s p}$, by the induced dipole moment of the cores [22]. In the following section, the results for spectroscopic constants at levels $A$ through $I$ are tabulated and discussed.

\section{Results and discussion}

\subsection{Atomic results}

As a first test, we apply our pseudopotentials and basis sets to the determination of atomic properties of atoms $X(X=\mathrm{In}, \mathrm{Sn}, \mathrm{Sb})$ and $Y(Y=\mathrm{F}, \mathrm{O}, \mathrm{S})$, ionization potentials (IP) and electron affinities (EA).

Our calculated values are given in table 1 at various levels of approximation using the frozen-core DF pseudopotentials with and without core-polarization terms. Our results are compared both to numerical all-electron and pseudopotential HF values [32] as well as to experimental ones. (With MCHF77 [32] numerical orbitals are employed and a comparison with all-electron calculations is possible, at

Table 1. Ionization potentials IP and electron affinities EA (in eV).

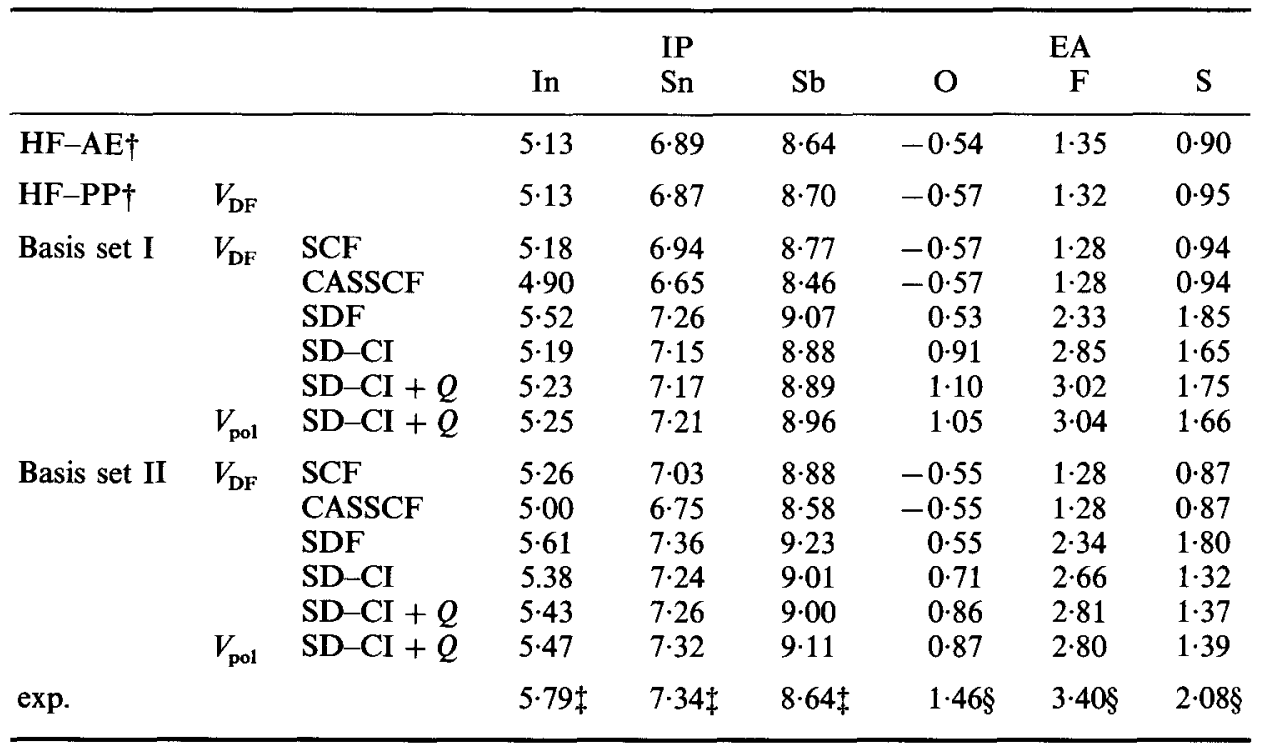

$\dagger$ Ref. [32]. $\ddagger$ Ref. [38]. § Ref. [39]. 
the HF level, without introducing basis set errors.) At the HF level, the pseudopotential errors for both ionization potentials and electron affinities, are smaller than $0.06 \mathrm{eV}$. With the exception of $\mathrm{Sn}$ and $\mathrm{F}$, the pseudopotential HF results are too large; this is mainly due to the overestimation of the valence exchange energy with radially nodeless pseudoorbitals. This error could be largely eliminated by improving our SEFIT (single-electron-fit) pseudopotentials with the MEFIT (multielectron-fit) procedure as already mentioned in [14]. The basis set errors arising when going from the numerical calculations to those using the GTO basis sets are $\leqslant 0.07 \mathrm{eV}$ (IP) and $\leqslant 0.04 \mathrm{eV}$ (EA) and up to $\sim 0.15 \mathrm{eV}$ (IP, EA) for basis sets I and II, respectively. Taking valence correlation into account, the behaviour of ionization potentials and electron affinities varies differently for the various methods used: with CASSCF (the active space is formed by the atomic valence orbitals), the ionization potential decreases by $\sim 0.3 \mathrm{eV}$ for $\mathrm{In}, \mathrm{Sn}$ and $\mathrm{Sb}$ with both basis sets. For $Y$ atoms $(Y=O, F, S)$, electron affinities are left unchanged, of course. With SDF, ionization potentials increase by $\sim 0.3 \mathrm{eV}$ with respect to SCF results. For electron affinities, the increase is much higher $(\sim 1 \mathrm{eV})$ due to the larger number of (paired) valence electrons. With SD-CI, ionization potentials grow by less than $0 \cdot 2 \mathrm{eV}$, whereas electron affinities are strongly affected: $\mathrm{F}$ shows the maximum increase $(\triangle E A \sim 1.5 \mathrm{eV})$ for both basis sets, while $\Delta E A$ is minimum in the case of $\mathrm{S}$ ( $\triangle E A=0.7 \mathrm{eV}$ and $0.5 \mathrm{eV}$ for basis set I and II, respectively). Contributions of quadruple excitations as estimated by means of the Davidson's correction [29] are nearly negligible in the case of IP $(\Delta \mathrm{IP} \leqslant 0.05 \mathrm{eV})$, but for electron affinities the increase is more important ( $\triangle \mathrm{EA} \leqslant 0.19 \mathrm{eV}$ for both basis sets). Going to the highest level of approximation (including core-valence correlation by means of $V_{\text {pol }}$, changes of ionization potentials and electron affinities are slight for both basis sets. The maximum effect is smaller than $0 \cdot 1 \mathrm{eV}$. Compared with experimental values $[38,39]$, ionization potentials and electron affinities are underestimated in most cases: with the exception of $\mathrm{Sb}$, where IP is overestimated by $0.3 \mathrm{eV}$ and $0.5 \mathrm{eV}$ for basis set I and II, respectively, ionization potentials are too small by $<0.5 \mathrm{eV}$. The defects are due neither to size-consistency nor to basis set deficiencies: on the one hand, both with CEPA-1 and multi-reference CI, similar results are obtained for both basis sets (IP $=5 \cdot 20 \mathrm{eV}$ and $5 \cdot 39 \mathrm{eV}$ for basis sets $\mathrm{I}$ and II, respectively); on the other hand, with an uncontracted large GTO basis set $(9 s 8 p 4 d)$, a SD-CI ionization potential of $5.41 \mathrm{eV}$ [24] results in excellent agreement with basis set II. Therefore, we conclude that, for In, these large deviations from the experimental ionization potential may be due to pseudopotential deficiencies, probably to the use of a dipole polarization potential, which underestimates polarization effects for the rather diffuse $d$ shells whereas it is quite adequate for $p$ shells [14]. In the case of $\mathrm{Sb}$, the experimental value might be unreliable as already mentioned in [14]. Electron affinities are underestimated by $\leqslant 0.4 \mathrm{eV}$ and $0.7 \mathrm{eV}$ for basis set I and II, respectively, because of difficulties in describing the $Y^{-}$ion $(Y=\mathrm{O}, \mathrm{F}, \mathrm{S})$ : basis set II contains only one $d$ function for correlating $p$ orbitals. For a more accurate description, functions of higher angular symmetry should be used. For oxygen, all-electron calculations have been performed by Feller and Davidson $[40,41]$. Their results with comparable basis set agree very well with ours [40]. Even with an extended $(11 s 6 p 4 d 2 f 1 g)$ / [8s5p4d2f1g] basis set, there still remains a deviation of $\sim 0.2 \mathrm{eV}$ from the experimental electron affinity [39]. Recently, SD-CI results with an extremely large uncontracted basis set $(23 s 12 p 10 d 5 f 3 g)$ and more than 5 million configurations have been published [41] reducing the deviation to the experimental value to $\sim 0 \cdot 1 \mathrm{eV}$. 
For fluorine, all-electron calculations using many-body perturbation theory [42] and the coupled cluster method [43] have been performed; in both cases, an electron affinity of $3.37 \mathrm{eV}$ is reported.

\subsection{Molecular results}

For the determination of the spectroscopic properties of the neutral $X_{2}, X \mathrm{H}$, $X \mathrm{O}, X \mathrm{~F}$ and $X \mathrm{~S}$ molecules $(X=\mathrm{In}, \mathrm{Sn}, \mathrm{Sb})$, all approximations $A$ to $I$ discussed in $\S 2$ have been considered. Our results for bond lengths $R_{e}$, dissociation energies $D_{e}$, vibrational frequencies $\omega_{e}$ and dipole moments $\mu_{e}$ are summarized in tables 2 to 7 , again at various levels of approximation, except for $\mu_{e}$, where only results at level $I$ are given.

Let us start with the discussion of bond lengths $R_{e}$. Bond lengths are only slightly changed by the approximations investigated in this work. The influence of core-core overlap can be judged from the first two columns $A$ and $B$ : it enlarges bond distances due to the repulsion of the charged cores, but the effects are nearly negligible $\left(\Delta R_{e} \leqslant 0.02\right.$ a.u. $)$, except for $\operatorname{InO}\left(\Delta R_{e}=0.13\right.$ a.u. $)$ and $\operatorname{SbO}\left(\Delta R_{e}=\right.$ 0.07 a.u.). Effects due to reducing our basis set are rather small, too, at the HF level: bond lengths are increased by $\leqslant 0.05$ a.u. for $X \mathrm{~F}(X=\mathrm{In}, \mathrm{Sn}, \mathrm{Sb})$ and $\leqslant 0.03$ a.u. for all other compounds, with the exception of $\mathrm{Sb}_{2}$, where $R_{e}$ is slightly shortened. When valence correlation is taken into account, the behaviour of the equilibrium distances is not unambigous: for CASSCF and SD-CI, $R_{e}$ becomes larger in most cases $\left(\Delta R_{e} \leqslant 0 \cdot 1\right.$ a.u.), whereas for SDF, it becomes shorter $\left(\Delta R_{e} \sim 0.03\right.$ a.u. $)$ due to the increasing value of the density functional with overlapping atomic densities. Exceptions are $\mathrm{In}_{2}$ (only in the case of CASSCF), InO and InS. For the fluorides

Table 2. Bond lengths $R_{e}$ (in a.u.), dissociation energies $D_{e}$ (in $\mathrm{eV}$ ) and vibrational frequencies $\omega_{e}$ (in $\left.\mathrm{cm}^{-1}\right)$ of $X_{2}(X=\mathrm{In}, \mathrm{Sn}, \mathrm{Sb}) . A, \mathrm{SCF} ; B$, same as $A$, but core-core interaction included; $C$, same as $B$, but basis set II; $D$, same as $C$, but CASSCF; $E$, same as $C$, but SDF; $F$, same as $C$, but SD-CI; $G$, same as $F$, but Davidson's correction included; $H$, same as $G$, but basis set $I ; I$, same as $G$, but $V_{\text {pol }}$ included; $K$, experimental value.

\begin{tabular}{|c|c|c|c|c|c|c|c|c|c|c|c|}
\hline & $X$ & $A$ & $B$ & $C$ & $D$ & $E$ & $F$ & $G$ & $H$ & $I$ & $K$ \\
\hline \multirow[t]{5}{*}{$R_{e}$} & In ${ }^{3} \Pi$ & $5 \cdot 84$ & 5.86 & $5 \cdot 89$ & 5.93 & 5.76 & 5.80 & 5.79 & $5 \cdot 78$ & $5 \cdot 70$ & - \\
\hline & ${ }^{3} \Sigma^{-}$ & - & - & - & - & - & - & - & - & $5 \cdot 27$ & $5.29 \dagger$ \\
\hline & ${ }^{1} \Sigma^{+}$ & - & - & - & - & - & - & - & - & $6 \cdot 31$ & - \\
\hline & $\operatorname{Sn}^{3} \Sigma^{-}$ & $5 \cdot 10$ & $5 \cdot 11$ & $5 \cdot 12$ & $5 \cdot 28$ & 5.08 & $5 \cdot 21$ & $5 \cdot 26$ & $5 \cdot 25$ & $5 \cdot 18$ & $5 \cdot 19 \ddagger$ \\
\hline & $\mathrm{Sb}^{1} \Sigma^{+}$ & 4.59 & $4 \cdot 60$ & $4 \cdot 59$ & $4 \cdot 82$ & $4 \cdot 55$ & $4 \cdot 69$ & $4 \cdot 75$ & $4 \cdot 76$ & $4 \cdot 70$ & $4 \cdot 70 \S$ \\
\hline \multirow[t]{5}{*}{$D_{e}$} & In ${ }^{3} \Pi$ & 0.51 & 0.51 & 0.53 & 0.77 & 0.93 & 0.77 & 0.89 & 0.92 & 1.09 & - \\
\hline & ${ }^{3} \Sigma-$ & - & - & - & - & 一 & - & - & - & 0.93 & $0.87 \dagger$ \\
\hline & ${ }^{1} \Sigma^{+}$ & - & - & - & - & - & - & - & - & 0.78 & - \\
\hline & $\operatorname{Sn}^{3} \Sigma^{-}$ & $1 \cdot 01$ & $1 \cdot 00$ & $1 \cdot 01$ & $1 \cdot 76$ & 1.99 & 1.49 & $1 \cdot 72$ & $1 \cdot 75$ & 1.90 & 2.009 \\
\hline & $\mathrm{Sb}^{1} \Sigma^{+}$ & -0.40 & -0.43 & -0.41 & 1.99 & 1.47 & $1 \cdot 14$ & 1.68 & 1.69 & 1.99 & $3 \cdot 11 \|$ \\
\hline \multirow[t]{5}{*}{$\omega_{e}$} & In ${ }^{3} \Pi$ & 109 & 109 & 111 & 107 & 122 & 118 & 117 & 112 & 117 & $115 \|$ \\
\hline & ${ }^{3} \Sigma^{-}$ & - & - & - & - & 一 & - & - & - & 136 & $142 \|$ \\
\hline & ${ }^{1} \Sigma^{+}$ & - & - & - & - & 一 & - & - & - & 88 & - \\
\hline & $\operatorname{Sn}^{3} \Sigma^{-}$ & 218 & 220 & 218 & 184 & 229 & 201 & 145 & 192 & 191 & $190 \ddagger$ \\
\hline & $\mathrm{Sb}^{1} \Sigma^{+}$ & 328 & 332 & 336 & 232 & 349 & 300 & 274 & 277 & 280 & $270 \|$ \\
\hline
\end{tabular}

† Ref. [46]. $\ddagger$ Ref. [47]. § Ref. [48, 49]. $\|$ Ref. [50]. T Ref. [51]. $a_{0}$ (a.u.) $\approx 5.2918 \times 10^{-11} \mathrm{~m}$. 


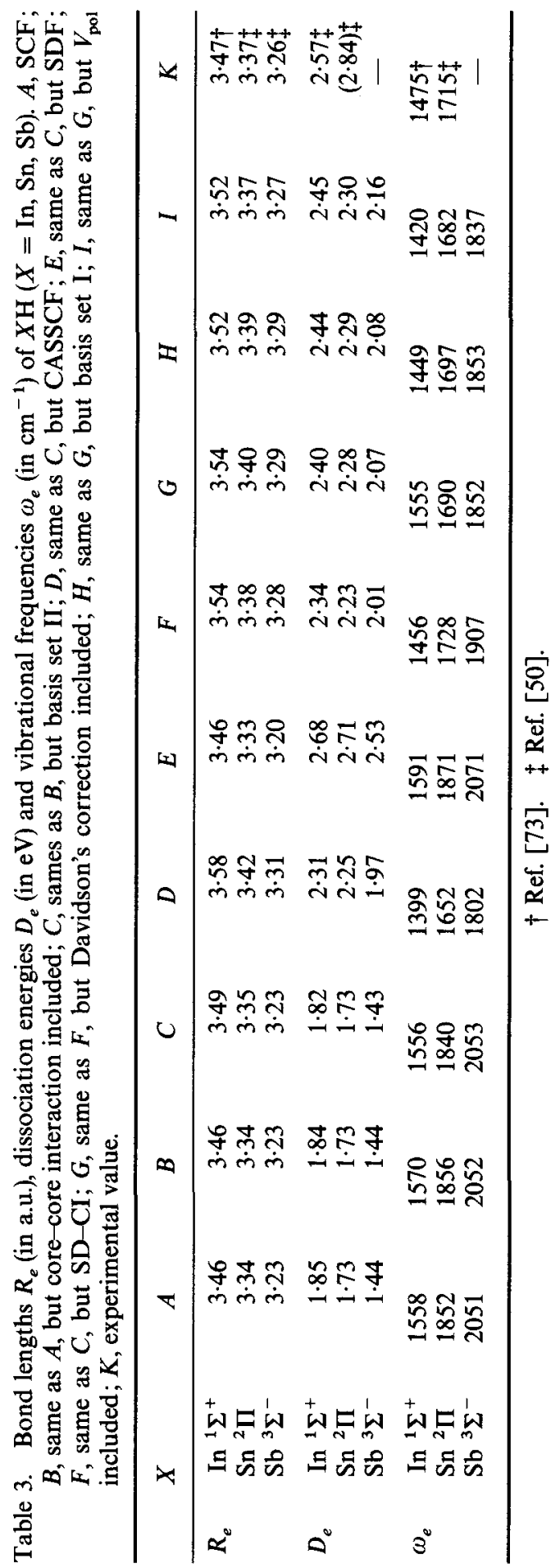




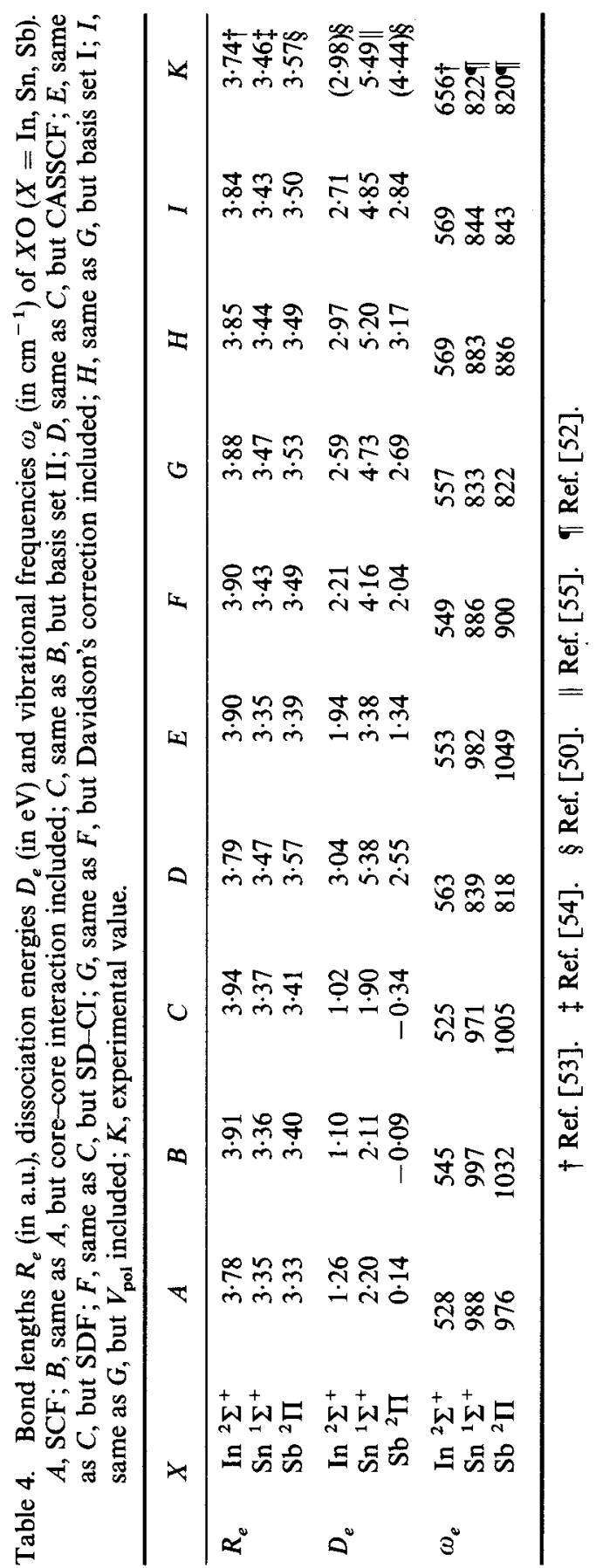


Table 5. Bond lengths $R_{e}$ (in a.u.), dissociation energies $D_{e}$ (in $\mathrm{eV}$ ) and vibrational frequencies $\omega_{e}$ (in $\left.\mathrm{cm}^{-1}\right)$ of $X \mathrm{~F}(X=\mathrm{In}, \mathrm{Sn}, \mathrm{Sb}) . A, \mathrm{SCF} ; B$, same as $A$, but core-core interaction included; $C$, same as $B$, but basis set II; $D$, same as $C$, but CASSCF; $E$, same as $C$, but SDF; $F$, same as $C$, but SD-CI; $G$, same as $F$, but Davidson's correction included; $H$, same as $G$, but basis set $I ; I$, same as $G$, but $V_{\text {pol }}$ included; $K$, experimental value.

\begin{tabular}{|c|c|c|c|c|c|c|c|c|c|c|c|}
\hline & $X$ & $A$ & $B$ & $C$ & $D$ & $E$ & $F$ & $G$ & $H$ & $I$ & $K$ \\
\hline$R_{e}$ & $\begin{array}{l}\text { In }{ }^{1} \Sigma^{+} \\
S^{2}{ }^{2} \Pi \\
S^{3}{ }^{3} \Sigma^{-}\end{array}$ & $\begin{array}{l}3.70 \\
3.60 \\
3.58\end{array}$ & $\begin{array}{l}3.71 \\
3.60 \\
3.58\end{array}$ & $\begin{array}{l}3.76 \\
3.64 \\
3.62\end{array}$ & $\begin{array}{l}3.81 \\
3.68 \\
3.67\end{array}$ & $\begin{array}{l}3 \cdot 73 \\
3 \cdot 61 \\
3 \cdot 58\end{array}$ & $\begin{array}{l}3.79 \\
3.68 \\
3.68\end{array}$ & $\begin{array}{l}3.80 \\
3.69 \\
3.69\end{array}$ & $\begin{array}{l}3.74 \\
3.63 \\
3.63\end{array}$ & $\begin{array}{l}3 \cdot 74 \\
3 \cdot 64 \\
3 \cdot 64\end{array}$ & $\begin{array}{l}3.75 \dagger \\
3.67 \dagger \\
3.62 \dagger\end{array}$ \\
\hline$D_{e}$ & $\begin{array}{l}\text { In }{ }^{1} \Sigma^{+} \\
\mathrm{Sn}^{2} \Pi^{+} \\
\mathrm{Sb}^{3} \Sigma^{-}\end{array}$ & $\begin{array}{l}3 \cdot 83 \\
3 \cdot 32 \\
2 \cdot 15\end{array}$ & $\begin{array}{l}3 \cdot 82 \\
3 \cdot 31 \\
2 \cdot 15\end{array}$ & $\begin{array}{l}3.69 \\
3.12 \\
1.93\end{array}$ & $\begin{array}{l}5 \cdot 24 \\
4 \cdot 39 \\
2 \cdot 66\end{array}$ & $\begin{array}{l}4 \cdot 57 \\
4.09 \\
2 \cdot 99\end{array}$ & $\begin{array}{l}4.79 \\
4.07 \\
2.94\end{array}$ & $\begin{array}{l}5.05 \\
4 \cdot 36 \\
3 \cdot 24\end{array}$ & $\begin{array}{l}5 \cdot 51 \\
4 \cdot 79 \\
3 \cdot 70\end{array}$ & $\begin{array}{l}5 \cdot 17 \\
4 \cdot 46 \\
3 \cdot 14\end{array}$ & $\begin{array}{c}5.28 \dagger \\
4.97 \dagger \\
(4.44) \dagger\end{array}$ \\
\hline$\omega_{e}$ & $\begin{array}{l}\text { In }{ }^{1} \Sigma^{+} \\
\operatorname{Sn}^{2} I^{-} \\
\text {sb }^{3} \Sigma^{-}\end{array}$ & $\begin{array}{l}541 \\
635 \\
653\end{array}$ & $\begin{array}{l}551 \\
638 \\
654\end{array}$ & $\begin{array}{l}523 \\
611 \\
615\end{array}$ & $\begin{array}{l}513 \\
590 \\
584\end{array}$ & $\begin{array}{l}545 \\
628 \\
662\end{array}$ & $\begin{array}{l}522 \\
591 \\
594\end{array}$ & $\begin{array}{l}516 \\
581 \\
581\end{array}$ & $\begin{array}{l}532 \\
610 \\
613\end{array}$ & $\begin{array}{l}534 \\
599 \\
616\end{array}$ & $\begin{array}{l}535 \dagger \\
583 \ddagger \\
610 \ddagger\end{array}$ \\
\hline
\end{tabular}

$\dagger$ Ref. [50]. $¥$ Ref. [52].

Table 6. Bond lengths $R_{e}$ (in a.u.), dissociation energies $D_{e}$ (in $\mathrm{eV}$ ) and vibrational frequencies $\omega_{e}$ (in $\left.\mathrm{cm}^{-1}\right)$ of $X \mathrm{~S}(X=\mathrm{In}, \mathrm{Sn}, \mathrm{Sb}) . A, \mathrm{SCF} ; B$, same as $A$, but core-core interaction included; $C$, same as $B$, but basis set II; $D$, same as $C$, but CASSCF; $E$, same as $C$, but SDF; $F$, same as $C$, but SD-CI; $G$, same as $F$, but Davidson's correction included; $H$, same as $G$, but basis set $I$; $I$, same as $G$, but $V_{\text {pol }}$ included; $K$, experimental value.

\begin{tabular}{|c|c|c|c|c|c|c|c|c|c|c|c|}
\hline & $X$ & $A$ & $B$ & $C$ & $D$ & $E$ & $F$ & $G$ & $H$ & $I$ & $K$ \\
\hline$R_{e}$ & $\begin{array}{c}\text { In }{ }^{2} \Sigma^{+} \\
\operatorname{Sn}^{1} \Sigma^{+} \\
\operatorname{Sb}^{2}{ }^{2} \Pi\end{array}$ & $\begin{array}{l}4 \cdot 39 \\
4 \cdot 08 \\
4 \cdot 09\end{array}$ & $\begin{array}{l}4 \cdot 41 \\
4 \cdot 08 \\
4 \cdot 09\end{array}$ & $\begin{array}{l}4 \cdot 44 \\
4 \cdot 09 \\
4 \cdot 10\end{array}$ & $\begin{array}{l}4 \cdot 31 \\
4 \cdot 19 \\
4 \cdot 25\end{array}$ & $\begin{array}{l}4 \cdot 18 \\
4 \cdot 06 \\
4 \cdot 06\end{array}$ & $\begin{array}{l}4 \cdot 28 \\
4 \cdot 14 \\
4 \cdot 16\end{array}$ & $\begin{array}{l}4 \cdot 31 \\
4 \cdot 16 \\
4 \cdot 20\end{array}$ & $\begin{array}{l}4 \cdot 29 \\
4 \cdot 14 \\
4 \cdot 17\end{array}$ & $\begin{array}{l}4 \cdot 28 \\
4 \cdot 12 \\
4 \cdot 17\end{array}$ & $\begin{array}{l}- \\
4 \cdot 17 \dagger\end{array}$ \\
\hline$D_{e}$ & $\begin{array}{l}\text { In }{ }^{2} \Sigma^{+} \\
\operatorname{Sn}^{1} \Sigma^{+} \\
\operatorname{Sb}^{2} \Pi\end{array}$ & $\begin{array}{l}1.60 \\
3.08 \\
0.97\end{array}$ & $\begin{array}{l}1.59 \\
3.08 \\
0.97\end{array}$ & $\begin{array}{l}1.49 \\
2.96 \\
0.81\end{array}$ & $\begin{array}{l}2 \cdot 67 \\
3 \cdot 70 \\
2 \cdot 50\end{array}$ & $\begin{array}{l}2 \cdot 51 \\
4 \cdot 40 \\
2 \cdot 42\end{array}$ & $\begin{array}{l}2 \cdot 30 \\
3.96 \\
1.99\end{array}$ & $\begin{array}{l}2 \cdot 59 \\
4 \cdot 34 \\
2 \cdot 46\end{array}$ & $\begin{array}{l}2.96 \\
4.60 \\
2.72\end{array}$ & $\begin{array}{l}2 \cdot 70 \\
4 \cdot 50 \\
2 \cdot 63\end{array}$ & $\begin{array}{c}(2 \cdot 95) \dagger \\
4 \cdot 80 \dagger \\
-\end{array}$ \\
\hline$\omega_{e}$ & $\begin{array}{l}\operatorname{In}^{2} \Sigma^{+} \\
\operatorname{Sn}^{1} \Sigma^{+} \\
\operatorname{Sb}^{2} \Pi\end{array}$ & $\begin{array}{l}164 \\
547 \\
551\end{array}$ & $\begin{array}{l}183 \\
552 \\
552\end{array}$ & $\begin{array}{l}244 \\
552 \\
550\end{array}$ & $\begin{array}{l}405 \\
487 \\
447\end{array}$ & $\begin{array}{l}246 \\
568 \\
568\end{array}$ & $\begin{array}{l}421 \\
527 \\
515\end{array}$ & $\begin{array}{l}405 \\
508 \\
694\end{array}$ & $\begin{array}{l}426 \\
515 \\
502\end{array}$ & $\begin{array}{l}370 \\
515 \\
488\end{array}$ & $\begin{array}{r}- \\
487 \dagger \\
480 \dagger\end{array}$ \\
\hline
\end{tabular}

$\dagger$ Ref. [50].

Table 7. Dipole moments $\mu_{e}$ (in D) of molecules $X Y(X=\mathrm{In}, \mathrm{Sn}, \mathrm{Sb} ; Y=\mathrm{H}, \mathrm{O}, \mathrm{F}, \mathrm{S})$. Experimental values in parentheses.

\begin{tabular}{|c|c|c|c|c|}
\hline $\begin{array}{ll}X & Y\end{array}$ & $\mathbf{H}$ & $\mathrm{O}$ & F & $\mathbf{S}$ \\
\hline In & 0.80 & $4 \cdot 06$ & $\begin{array}{c}3.59 \\
(3.40 \pm 0.07) \dagger\end{array}$ & $4 \cdot 21$ \\
\hline $\mathrm{Sn}$ & 0.66 & $\begin{array}{c}4 \cdot 67 \\
(4 \cdot 32 \pm 0 \cdot 1) \dagger\end{array}$ & 2.94 & $\begin{array}{c}3 \cdot 75 \\
(3 \cdot 18 \pm 0 \cdot 1) \dagger\end{array}$ \\
\hline $\mathrm{Sb}$ & $0 \cdot 26$ & 3.97 & $3 \cdot 15$ & $2 \cdot 70$ \\
\hline
\end{tabular}

$\dagger$ Ref. [56]. 
$X F(X=\mathrm{In}, \mathrm{Sn}, \mathrm{Sb}), \mathrm{CASSCF}$ and SD-CI results are nearly equivalent. Contributions of quadruple excitations to the equilibrium distances are small: $R_{e}$ is normally increased by $\leqslant 0.06$ a.u., except for $\mathrm{In}_{2}$ and $\mathrm{InO}$ where the bond length is shortened by 0.01 a.u. and 0.02 a.u., respectively. At this level, comparison of basis sets I and II is possible again: the results deviate by $\leqslant 0.06 \mathrm{a}$.u. The highest level of approximation is reached by taking core-valence correlation into account: in all cases, bond lengths decrease by $<0 \cdot 1$ a.u. compared with SD-CI $+Q$ results (columns $G$ and $I$ in the tables). At this level of approximation, comparison with experimental values is possible. Unfortunately, for the sulphides $X S(X=\mathrm{In}, \mathrm{Sn}, \mathrm{Sb})$, few experimental values of $R_{e}$ are known. In the case where reliable experimental data are available, our calculated bond lengths are underestimated by $\leqslant 0.07$ a.u., with the exception of $\operatorname{InO}\left(\Delta R_{e}=+0 \cdot 1\right.$ a.u. $)$ and $\operatorname{SbF}\left(\Delta R_{e}=+0.02\right.$ a.u. $)$. This error may be due, in part, to the overestimation of valence correlation energy when using nodeless pseudoorbitals $[44,45]$.

We now turn to dissociation energies $D_{e}$. With the exception of $\mathrm{Sb}_{2}$, we found all molecules to be stable even at the SCF level. Dissociation energies become smaller by taking core-core overlap into account, in accordance with the increase of bond lengths. The effect is largest for the oxides $X O(X=\mathrm{In}, \mathrm{Sn}, \mathrm{Sb})\left(\Delta D_{e} \leqslant 0 \cdot 2 \mathrm{eV}\right)$, while it is negligible for all other molecules investigated in this paper. $\left(D_{e}\right.$ of the sulphides $X \mathrm{~S}(X=\mathrm{In}, \mathrm{Sn}, \mathrm{Sb})$ is less affected by core-core interaction than that of the corresponding oxides because of the larger binding distances.) With the smaller basis set II, $0 \cdot 1$ to $0.2 \mathrm{eV}$ are lost in dissociation energies, at the HF level, with respect to basis set $\mathrm{I}$. (Thus, for $\mathrm{SbO}, D_{e}$ becomes negative.) Valence correlation is the largest contribution to dissociation energies. The largest increase of $D_{e}$ due to valence correlation is observed in the case of the oxides for CASSCF, SDF and SD-CI. For dimers $X_{2}$ and hydrides $X H(X=\mathrm{In}, \mathrm{Sn})$, all three correlation methods used in this work yield nearly the same dissociation energies. Generally, the SD-CI results (level $F$ ) become worse with increasing number of valence electrons because of the concomitant increase of size-consistency errors; for SbF, e.g. only 66 per cent of the experimental dissociation energy is obtained, whereas for InH nearly 100 per cent is reached. Accordingly, contributions of quadruple excitations become more and more important with increasing number of electrons $\left(\Delta D_{e}=0.05 \mathrm{eV}\right.$ and $0 \cdot 30 \mathrm{eV}$ for InH and SbF, respectively). Comparing basis sets I and II, the differences are $\leqslant 0.04 \mathrm{eV}$ for dimers $X_{2}$ and hydrides $X \mathrm{H}$, but increase for oxides $X \mathrm{O}$, fluorides $X F$ and sulphides $X S(X=\mathrm{In}, \mathrm{Sn}, \mathrm{Sb})$ due to difficulties in describing the electron affinities of $Y(Y=O, F, S)$ with basis set II. For the oxides and fluorides, dissociation energies obtained with basis set II, are by $\sim 0.5 \mathrm{eV}$ smaller than those with basis set $I$ (cf. levels $G$ and $H$ in the tables) whereas for the sulphides, the deviations are slightly smaller $(\leqslant 0.4 \mathrm{eV})$ probably due to the smaller electronegativity of $S$. With basis set $I$, the deviations from experimental data (without taking core-valence correlation into account) are smaller than $0.3 \mathrm{eV}$, except for $\mathrm{SbF}$; this indicates that, for CI calculations, basis set II seems to be too small. Core-valence correlation contributes about $0 \cdot 15 \mathrm{eV}$ to dissociation energies with the exception of the dimers $X_{2}$ and the hydrides $X H(X=\mathrm{In}, \mathrm{Sn}, \mathrm{Sb})$ : for the dimers, $\Delta D_{e}$ is at a maximum $(\leqslant 0 \cdot 3 \mathrm{eV})$ whereas, for the hydrides, $\Delta D_{e}$ is minimal $(\leqslant 0 \cdot 1 \mathrm{eV})$. Comparison with experimental dissociation energies can now be made at this level of approximation (I): as already mentioned above, our calculated dissociation energies underestimate the experimental ones where available mainly due to the use of the small basis set II which seems to be insufficient for CI calculations. (Unfortunately, experimental 
values become rather rare for the sulphides $X \mathrm{~S}$ with $X=\mathrm{In}, \mathrm{Sn}$ and $\mathrm{Sb}$.) The deviations increase from In $Y$ to $\operatorname{Sb} Y(Y=X, \mathrm{H}, \mathrm{O}, \mathrm{F}, \mathrm{S})$ because of the increasing number of valence electrons, which have to be correlated. Size-consistency errors seem to be not very large: e.g. for $\mathrm{SbF}$ and $\mathrm{InO}$, the deviations of our $\mathrm{SD}-\mathrm{CI}+Q$ results from CEPA-1 results are $0.06 \mathrm{eV}$ and $\sim 0.3 \mathrm{eV}$, respectively, for both basis sets. (We choose these examples because for InO, the coefficient of the SCF reference determinant is at a minimum $(0.85)$ and for $\mathrm{SbF}$, the number of valence electrons is largest.)

The discussion of vibrational frequencies $\omega_{e}$ can be dealt with briefly because their behaviour is closely connected to that of $R_{e}$ discussed above. Due to the core overlap correction, $\omega_{e}$ slightly increases. Changing from basis set I to basis set II leads to a decrease in most cases; exceptions are found for some In and $\mathrm{Sb}$ compounds $\left(\mathrm{In}_{2}, \mathrm{Sb}_{2}, \mathrm{SbH}\right.$ and $\left.\mathrm{InO}\right)$. With few exceptions, $\omega_{e}$ is decreased by valence correlation using CASSCF or SD-CI, but when SDF is used an opposite tendency is found for all molecules considered here; this is related to the underestimation of bond length with SDF. With the exception of $\mathrm{Sn}_{2}$ and $\mathrm{InH}$, vibrational frequencies are slightly affected by the change of basis set at $\mathrm{CI}$ level. Core-valence correlation enlarges $\omega_{e}$ in accordance with the decrease of bond lengths, except in the case of the hydrides $\mathrm{InH}, \mathrm{SnH}$ and $\mathrm{SbH}$. Our calculated vibrational frequencies agree very well with experimental values; the deviation is only $\sim 30 \mathrm{~cm}^{-1}$, with the exception of InH and InO, where $\Delta \omega_{e}$ is $56 \mathrm{~cm}^{-1}$ and $87 \mathrm{~cm}^{-1}$, respectively. For $\mathrm{InH}, \mathrm{SnH}$ and InO, the experimental $\omega_{e}$ are underestimated, whereas the opposite is true for the other compounds.

Let us now turn to the ground state of $\operatorname{In}_{2}$ where experimental investigations on $\mathrm{In}_{2}[46,57]$ led to conflicting results: Douglas et al. [57] found the ${ }^{1} \Sigma^{+}$state to be the most stable one, whereas Froben et al. [46] reported a ${ }^{3} \Sigma^{-}$ground state. All-electron calculations performed by Balasubramanian and $\mathrm{Li}$ [58], however, yielded a ${ }^{3} \Pi$ ground state. Therefore, we have investigated these three low-lying states of $\operatorname{In}_{2}$. As shown in table 2, we found the ${ }^{3} \Pi$ state to be the ground state in agreement with Balasubramanian and $\mathrm{Li}$ [58]. The ${ }^{1} \Sigma^{+}$state is higher in energy by $0.15 \mathrm{eV}$ than the ${ }^{3} \Sigma^{-}$state which lies $0.16 \mathrm{eV}$ above the ground state. Our calculated bond length and dissociation energy of the ${ }^{3} \Sigma^{-}$state are in excellent agreement with the experimental values determined by Froben et al. [46]: the deviations are 0.02 a.u. $\left(R_{e}\right)$ and $0.06 \mathrm{eV}\left(D_{e}\right)$.

Additionally, investigation is made on dipole moments $\mu_{e}$ at the highest level of approximation $(I)$. Our calculated values are corrected by the induced dipole moment [22] of the $X$ cores ( $X=\mathrm{In}, \mathrm{Sn}, \mathrm{Sb})$. We have collected our results in table 7 and have compared with experimental data where available. In all cases where comparison is possible, our calculated dipole moments are consistently too large. The deviation is $<0.6 \mathrm{D}$. For the hydrides $X \mathrm{H}$ and for the sulphides $X \mathrm{~S}$ ( $X=\mathrm{In}$, $\mathrm{Sn}, \mathrm{Sb})_{,} \mu_{e}$ decreases with increasing number of valence electrons of atom $X$. In the case of $\mathrm{Sn}$ and $\mathrm{Sb}$ compounds, a maximum of $\mu_{e}$ is reached at the oxides.

We now turn to comparison of our results with all-electron or pseudopotential calculations by other authors. Due to the computational effort and difficulties to describe relativistic effects correctly, mostly pseudopotential or $X_{\alpha}$ results have been published [58-71]. Only for $\mathrm{SnH}$ [68], ab initio all-electron calculations have been performed. To our knowledge, papers have appeared on $\operatorname{In}_{2}$ [58], InH [59], InO $[60], \mathrm{Sn}_{2}[61-65], \mathrm{SnH}[66-68], \mathrm{SnO}[69,70]$, and $\mathrm{SbH}[71]$. For fluorides $X \mathrm{~F}$ and sulphides $X S$ ( $X=$ In, Sn, Sb), no theoretical investigations exist. Let us start with 
In compounds. For $\mathrm{In}_{2}$, Balasubramanian and $\mathrm{Li}[58]$ found the ${ }^{3} \Pi$ as ground state in agreement with our results as already mentioned above. But their calculated $R_{e}$ is 0.23 a.u. larger than ours, whereas their $D_{e}$ is $0.26 \mathrm{eV}$ smaller. For $\mathrm{InH}$, our results are in excellent agreement for $R_{e}, D_{e}$ and $\omega_{e}$ with those of Teichteil and Spiegelmann [59]. For InO, $X_{\alpha}$ calculations have been performed [60]; an equilibrium bond distance of $3.66 \mathrm{a}$.u. and a vibrational frequency of $764 \mathrm{~cm}^{-1}$ are reported, which deviate from our results by $\sim 0 \cdot 2$ a.u. $\left(R_{e}\right)$ and $200 \mathrm{~cm}^{-1}\left(\omega_{e}\right)$, respectively. We now turn to molecules containing $\mathrm{Sn}$. For $\mathrm{Sn}_{2}$, Andzelm et al. [61] obtained excellent agreement both with experimental and our results for bond length and vibrational frequency using model potentials and local exchangecorrelation spin density functionals (LSD), but the dissociation energy is overestimated by $1 \mathrm{eV}$ as usual with exchange-correlation LSD. Relativistic effective potentials are used by Balasubramanian and Pitzer [62-64]; the results show similar deviations to experimental values as ours. $R_{e}$ and $\omega_{e}$ are overestimated by $\sim 0.1$ a.u. and $\sim 10 \mathrm{~cm}^{-1}$, respectively, by Pacchioni [65]. For $\mathrm{SnH}$, other theoretical investigations using pseudopotentials [66-68] agree very well with our results for $R_{e}$ and $\omega_{e}\left(\Delta R_{e} \sim 0.05\right.$ a.u., $\left.\Delta \omega_{e} \sim 10 \mathrm{~cm}^{-1}\right)$, but the dissociation energy is by $0.3 \mathrm{eV}$ smaller than ours $[66,67]$ and by $0.8 \mathrm{eV}$ smaller than the experimental value, respectively. Therefore, the authors conclude that the experimental $D_{e}$ might be unreliable [67]. Pettersson and Langhoff [68] obtained a dipole moment of SnH of $\sim 0.4 \mathrm{D}$ by means of all-electron calculations in agreement with pseudopotential calculations [66]. For SnO, SD-CI calculations of Balasubramanian and Pitzer [69] using relativistic effective potentials overestimate $R_{e}$ by $0 \cdot 2$ a.u. and their dissociation energy is by $\sim 1.8 \mathrm{eV}$ too small. These deviations both with experimental data and with our results are mainly due to basis set deficiencies: the oxygen basis set of Balasubramanian and Pitzer [69] should be augmented by diffuse $p$ and $d$ exponents to describe correctly the oxygen anion $\mathrm{O}^{-}$as outlined in [40] and [41]. $X_{\alpha}$ calculations [70] are rather successful in describing the bond length and vibrational frequency of $\mathrm{SnO}$ : values of $3.46 \mathrm{a} . \mathrm{u}$. and $822 \mathrm{~cm}^{-1}$ for $R_{e}$ and $\omega_{e}$, respectively, are obtained. For $\mathrm{SbH}$, calculations using relativistic effective potentials [71] lead to a bond length which is $\sim 0.16 \mathrm{a}$.u. larger than both the experimental and our value; the calculated dissociation energy is $2 \cdot 26 \mathrm{eV}$.

\section{Conclusion}

We have investigated ground-state properties of dimers, hydrides, oxides, fluorides and sulphides of $\mathrm{In}, \mathrm{Sn}$ and $\mathrm{Sb}$ using pseudopotentials. The results have been discussed at various levels of approximation. It has been shown that both core-core overlap correction and core-polarization effects are rather small for the molecules investigated. (Only in the case of the dimers $X_{2}$, core-valence correlation plays a significant role.) Therefore, both effects can be neglected in determinations of geometries and stabilities of associations $\left(X Y_{m}\right)_{n}(X=\mathrm{In}, \mathrm{Sn}, \mathrm{Sb} ; Y=\mathrm{H}, \mathrm{O}, \mathrm{F}, \mathrm{S} ; n \leqslant 6)$ of these molecules which will be published in a forthcoming paper [72]. The quality of two basis sets is discussed both at HF and CI level. We have shown that, at the HF level, results obtained with basis set II are in good agreement with those obtained with the larger basis set I. At the CI level, however, larger deviations result. As we only intend to qualitatively investigate the associations $\left(X Y_{m}\right)_{n}$ at the HF level [72], we conclude that basis set II seems to be sufficient for this purpose. Furthermore, several methods of valence correlation treatment have been taken into account. 
SD-CI and SDF results are of comparable accuracy. Therefore, for qualitative investigations on larger molecules, SDF will be performed.

At the highest level of approximation, both valence correlation and core-valence correlation are taken into account by means of SD-CI with Davidson's correction and core-polarization potentials with electron-electron and electron-other core terms, respectively. Compared to reliable experimental data where available, the deviations are less than $0.06 \AA\left(R_{e}\right), 0.3 \mathrm{eV}\left(D_{e}\right), 87 \mathrm{~cm}^{-1}\left(\omega_{e}\right)$ for basis set I and $0.05 \AA\left(R_{e}\right), 0.6 \mathrm{eV}\left(D_{e}\right), 87 \mathrm{~cm}^{-1}\left(\omega_{e}\right)$ for basis set II, respectively. Dipole moments $\mu_{e}$ are accurate up to $0.6 \mathrm{D}$. The errors are maximum in the case of the oxides $X O$ $\left(X=\mathrm{In}, \mathrm{Sn}, \mathrm{Sb}\right.$ ) because of difficulties in describing the $\mathrm{O}^{-}$ion. For $\mathrm{Sb}$ compounds, the deviations are larger than for In and Sn compounds. This is mainly due to the pseudopotential adjustment. (Note, that the Sb pseudopotential had been adjusted to DF energies because of the insufficient number of experimental data.) Our results agree very well with those of other theoretical investigations where present. To our knowledge, spectroscopic properties of fluorides and sulphides of In, $\mathrm{Sn}$ and $\mathrm{Sb}$ as well as of $\mathrm{SbO}$ have not been calculated up to now. Predictions for experimental unknown or unreliable properties have been made. The ground state of $\operatorname{In}_{2}$ has been found to be ${ }^{3} \Pi$ in contrast to experimental results but in agreement with other relativistic calculations [58].

Thanks are due to U. Wedig and M. Dolg for implementing the programs used in this work on the CRAY-2 in Stuttgart and to $\mathbf{H}$. Hayd and M. Kohout for valuable discussions. The financial support of the Deutsche Forschungsgemeinschaft is gratefully acknowledged.

\section{References}

[1] Weeks, J. D., Hazi, A., and Rice, S. A., 1969, Adv. chem. Phys., 16, 283.

[2] Bardsley, J. N., 1974, Case Stud. At. Phys., 4, 299.

[3] Dixon, R. N., and Rober TSON, I. L., 1978, Specialist Report on Theoretical Chemistry, Vol. 3 (The Chemical Society).

[4] Krauss, M., and Stevens, W. J., 1984, A. Rev. phys. Chem., 35, 357.

[5] Christiansen, P. A., Ermler, W. C., and Pitzer, K. S., 1985, A. Rev. phys. Chem., 36, 407.

[6] PyYkxö, P., 1986, Relativistic Theory of Atoms and Molecules, A Bibliography 19161985 (Springer).

[7] KutzelnigG, W., 1987, Phys. scripta, 36, 416.

[8] WADT, W. R., and HAY, P. J., 1985, J. chem. Phys., 82, 284.

[9] La John, L. A., Christiansen, P. A., Ross, R. B., Atashroo, T., and Ermler, W. C., 1987, J. chem. Phys., 87, 2812.

[10] Teichteil, C., Pelissier, M., and Spiegelmann, F., 1983, Chem. Phys., 81, 273.

[11] Andzelm, J., Radzio, E., and Salahub, D. R., 1985, J. chem. Phys., 83, 4573.

[12] Christiansen, P. A., Balasubramanian, K., and Pitzer, K. S., 1982, J. chem. Phys., 76, 5087.

[13] Hurley, M. M., Pacios, L. F., Christiansen, P. A., Ross, R. B., and Ermler, W. C., 1986, J. chem. Phys., 84, 6840.

[14] Igrl-Mann, G., Stoll, H., and Preuss, H., 1988, Molec. Phys., 65, 1321.

[15] Preuss, H., Stoll, H., Wedig, U., and KrÜGer, Th., 1981, Int. J. quant. Chem., 19, 113.

[16] Müller, W., Flesch, J., and Meyer, W., 1984, J. chem. Phys., 80, 3297.

[17] Wedig, U., Stoll, H., and Preuss, H., 1981, Chem. Phys., 61, 117.

[18] Fuentealba, P., Preuss, H., Stoll, H., and Szentpály, L. V., 1982, Chem. Phys. Lett., $89,418$.

[19] Szentpály, L. v., Fuentealba, P., Preuss, H., and Stoll, H., 1982, Chem. Phys. Lett., 93, 555 . 
[20] Flad, J., Igel, G., Dolg, M., Stoll, H., and Preuss, H., 1983, Chem. Phys., 75, 331.

[21] Schwerdtfeger, P., Szentpály, L. v., Vogel, K., Silberbach, H., Stoll, H., and Preuss, H., 1986, J. chem. Phys., 84, 1606.

[22] Igel-ManN, G., Wedig, U., Fuentealba, P., and Stoll, H., 1986, J. chem. Phys., 84, 5007.

[23] Savin, A., Vogel, K., Preuss, H., Stoll, H., Nesper, R., and Schnering, H.-G. V., 1988, J. Am. chem. Soc., 110, 373.

[24] Igel-ManN, G., Stoll, H., and Preuss, H., 1988, Molec. Phys., 65, 1329.

[25] Plass, W., Savin, A., Stoll, H., Preuss, H., Nesper, R., and Schnering, H.-G. v., Inorg. Chem. (submitted).

[26] Stoll, H., Fuentealba, P., Dolg, M., Flad, J., Szentpály, L. V., and Preuss, H., 1983, J. chem. Phys., 79, 5532.

[27] Werner, H.-J., University of Bielefeld, MEYer, W., University of Kaiserlautern, 1987, program MOLPRO.

[28] MCMurchie, L., Elbert, S., Langhoff, S., Davidson, E. R., 1982, program MELD, Washington University, Seattle.

[29] LanghofF, S., and Davidson, E. R., 1974, Int. J. quant. Chem., 8, 61.

[30] HAYD, H. (unpublished results).

[31] Barthelat, J. C., Pelissier, M., Villemur, P., and Devilliers, R., 1981, program PSATOM, University of Toulouse.

[32] Froese FisCHER, C., 1977, program MCHF77, Pennsylvania State University. Dolg, M., 1987, modified version of MCHF77, University of Stuttgart.

[33] Wood, J. H., and Boring, A. M., 1978, Phys. Rev. B, 18, 2701.

[34] Stoll, H., Pavlidou, C. M. E., and Preuss, H., 1978, Theor. chim. Acta, 49, 143.

[35] Stoll, H., Golka, E., and Preuss, H., 1980, Theor. chim. Acta, 55, 28.

[36] Perdew, J. P., 1986, Phys. Rev. B, 33, 8822.

[37] Lee, C., YANG, W., and PARR, R. G., 1988, Phys. Rev. B, 37, 785.

[38] MoOre, C. E., 1949, 1952, 1958, Atomic Energy Levels, Natl. Bur. Stand. Circ. No. 467 (U.S. GPO, Washington, D.C.), Vols. 1-3.

[39] НоTop, H., and Lineberger, W. C., 1975, J. Phys. Chem. Ref. Data, 4, 539.

[40] Feller, D., and Davidson, E. R., 1985, J. chem. Phys., 82, 4135.

[41] Feller, D., and Davidson, E. R., 1989, J. chem. Phys., 90, 1024.

[42] Ortiz, J. V., 1987, J. Chem. Phys., 86, 308.

[43] Adamowicz, L., and Bartlett, R. J., 1986, J. chem. Phys., 84, 6837.

[44] Fuentealba, P., Szentpály, L. V., Preuss, H., and Stoll, H., 1985, J. Phys. B, 18, 1287.

[45] Pittel, B., and Schwarz, W. H. E., 1977, Chem. Phys. Lett., 46, 121.

[46] Froben, F. W., Schulze, W., and Kloss, U., 1983, Chem. Phys. Lett., 99, 500.

[47] Bondybey, V. E., Heaven, M., and Miller, T. A., 1983, J. chem. Phys., 78, 3593.

[48] Gerber, G., and Kuscher, G., 1981, Chem. Phys., 60, 119.

[49] Sontag, H., and Weber, R., 1982, Chem. Phys., 70, 23.

[50] Huber, K. P., and HerzberG., G., 1979, Molecular Spectra and Molecular Structure, Vol. IV (Van Nostrand).

[51] Epting, M. E., McKenzie, M. T., and Nixon, E. R., 1980, J. chem. Phys., 73, 134.

[52] Radzig, A. A., and Smirnov, B. M., 1985, Reference Data on Atoms, Molecules and Ions (Springer).

[53] Jacquinot, D., and Lavendy, H., 1975, C. r. hebd. Séanc. Acad. Sci., Paris B, 281, 397.

[54] Appelblad, O., Fredin, S., and LagerQvist, A., 1984, Phys. scripta, 29, 561.

[55] LaU, K. H., Brittain, R. D., and Hildenbrand, D. L., 1981, Chem. Phys. Lett., 81, 227.

[56] Landolt-BörnsteIn, 1974, Molecular Constants, Vol. II/6 (Springer).

[57] Douglas, M. A., Hauge, R. H., and Margrave, J. L., 1983, J. phys. Chem., 87, 2945.

[58] Balasubramanian, K., and Li, J., 1988, J. chem. Phys., 88, 4979.

[59] Teichteil, C., and Spiegelmann, F., 1983, Chem. Phys., 81, 283.

[60] KovBA, V. M., and Topol, I. A., 1986, J. molec. Struct. (Theochem.), 137, 65.

[61] Andzelm, J., Russo, N., and Salahub, D. R., 1987, J. chem. Phys., 87, 6562.

[62] Balasubramanian, K., and Pitzer, K. S., 1983, J. chem. Phys., 78, 321.

[63] Balasubramanian, K., and Pitzer, K. S., 1984, J. chem. Phys., 80, 592.

[64] Pitzer, K. S., 1984, Int. J. quant. Chem., 25, 131.

[65] Pacchioni, G., 1985, Molec. Phys., 55, 211. 
[66] Chapman, D. A., Li, J., Balasubramanian, K., and Lin, S. H., 1988, J. chem. Phys., 88, 3826.

[67] Balasubramanian, K., and Pitzer, K. S., 1984, J. molec. Spectrosc., 103, 105.

[68] Pettersson, L. G. M., and Langhoff, S. R., 1986, Chem. Phys. Lett., 125, 429.

[69] Balasubramanian, K., and Pitzer, K. S., 1983, Chem. Phys. Lett., 100, 329.

[70] Dyke, J. M., Morris, A., Ridha, A. M. A., and Snidders, J. G., 1982, Chem. Phys., 67, 245.

[71] Balasubramanian, K., Tanpipat, N., and Bloor, J. E., 1987, J. molec. Spectrosc., 124, 458.

[72] Igel-Mann, G., Flad, H.-J., Feller, C., Savin, A., Stoll, H., and Preuss, H. (to be published).

[73] Bahnmaier, A. H., Urban, R.-D., and Jones, H., 1989, Chem. Phys. Lett., 155, 269. 